\title{
A Coupled Smoothed Finite Element-Boundary Element Method for Structural-Acoustic Analysis of Shell
}

\author{
Wanyi TIAN ${ }^{(1)}$, Lingyun $\mathrm{YAO}^{(2)}, \mathrm{Li} \mathrm{LI}^{(2)}$ \\ (1) Modern Engineering Training Center \\ Hunan University \\ Changsha, 410008, China \\ (2) College of Engineering and Technology \\ Southwest University \\ Chongqing, 400715, P.R. China; e-mail: 19831022y@163.com \\ (received August 28, 2015; accepted October 19, 2016)
}

\begin{abstract}
Nowadays, the finite element method (FEM) - boundary element method (BEM) is used to predict the performance of structural-acoustic problem, i.e. the frequency response analysis, modal analysis. The accuracy of conventional FEM/BEM for structural-acoustic problems strongly depends on the size of the mesh, element quality, etc. As element size gets greater and distortion gets severer, the deviation of high frequency problem is also clear. In order to improve the accuracy of structural-acoustic problem, a smoothed finite-element/boundary-element coupling procedure (SFEM/BEM) is extended to analyze the structural-acoustic problem consisting of a shell structure interacting with the cavity in this paper, in which the SFEM and boundary element method (BEM) models are used to simulate the structure and the fluid, respectively. The governing equations of the structural-acoustic problems are established by coupling the SFEM for the structure and the BEM for the fluid. The solutions of SFEM are often found to be much more accurate than those of the FEM model. Based on its attractive features, it was decided in the present work to extend SFEM further for use in structural-acoustic analysis by coupling it with BEM, the present SFEM/BEM is implemented to predict the vehicle structure-acoustic frequency response analysis, and two numerical experiments results show that the present method can provide more accurate results compared with the standard FEM/BEM using the same mesh. It indicates that the present SFEM/BEM can be widely applied to solving many engineering noise, vibration and harshness $(\mathrm{NVH})$ problems with more accurate solutions.
\end{abstract}

Keywords: smoothed finite element method (SFEM); FEM; BEM; structural-acoustic analysis.

\section{Introduction}

The interaction between the vibrating structure and the acoustic fluid field can, in many engineering noise, vibration and harshness (NVH) analyses, significantly affect the response of the structure and hence needs to be taken into account properly. Numerical analysis of shell structural-acoustic problem is a complex task, involving the modeling of both the structure domain, the acoustic fluid domain, and the interaction between these two domains, which requires proper treatment of the sub-domains in which different physical phenomena are involved, as well as suitable numerical modeling of wave propagation across interfaces. Displacement is generally the primary vari- able that can be used to describe structural behavior, while, in the acoustic fluid domain, several different primary variables for modeling the fluid field have been proposed, e.g., displacement (HAMDI et al., 1978), potential (Everstine, 1981; Morand, OHAYOn, 1979), pressure, and the combination of some of these variables (BATHE et al., 1995). In this paper, the acoustic pressure in fluid coupled with the displacement in the structure domain is adopted to describe the structuralacoustic problems.

Numerous researchers have studied many different numerical methods for use in solving structuralacoustic problems. The finite element method (FEM) is a favorable numerical approach for engineering problems, including structural-acoustic problems 
(DAvidsson, 2004; Everstine, 1997). The wellknown problems of using fluid elements in the finite element method (FEM) are the need for an approximate radiation boundary condition at the outer fluid boundary, limitations on mesh size and type, and the difficulty of generating the fluid mesh.

The boundary element method (BEM) has been widely used to model fluids. It is an attractive technique for the analysis of structural-acoustic problem since only the surfaces need to be discretized In addition, the BEM offers the advantage in the discretization compared with the FEM. In order to take advantage of both the FEM and the BEM, coupled finite element method/boundary element method (FEM/BEM) approaches have been proposed (EVERSTINE, HENDERSON, 1990; JeAns, Mathews, 1990; 1993). The coupled finite element method/boundary element method (FEM/BEM) is a conventional means of computing responses for fluid-structure interaction system (KoPUz, 1996; TONGA et al., 2007) and structural-acoustic problem (Choi, 1997; Chen et al., 2000; Coyette, 1999). As is well known, the coupled FEM/BEM is a conventional means of solving the structural-acoustic problem.

The results of coupled FEM/BEM become quite inaccurate because of the "overly-stiff" property of FEM (LIU et al., 2007b). Therefore, the coupled FEM/BEM models also possess the "overly-stiff" property due to the use of FEM model. The smoothed finite-element method (SFEM) (LIU et al., 2007a; WANG et al., 2015; LI et al., 2014; HE et al., 2011; 2013) was recently proposed by incorporation of the gradient smoothing technique with the standard finite element techniques. The primary variable and its gradient solutions of SFEM are often found to be much more accurate than those of the FEM model using quadrilateral meshes. Owning to the "properly-stiff" property of SFEM model, it was decided in the present work to extend SFEM further for use in structural-acoustic analysis by coupling it with BEM. Thus, the authors have formulated a coupled SFEM/BEM for addressing problems associated with structural-acoustic coupled systems that are described by the displacement in the structural domain coupled with the acoustic pressure in the fluid domain. Exclusion of the cell-based gradient smoothing operation was done on the shell element in SFEM, and the formulations of SFEM/BEM are very general and applicable to structural-acoustic problems. According to the previous research it is natural to expect that the SFEM/BEM will obtain more accurate results than FEM/BEM for structure-acoustic problems, even for the vehicle structure-acoustic coupled problems. The numerical results shown that the present method can be well applied to solving vehicle acoustic model with more accurate solutions. The investigation indicates the proposed method has great potential in the practical analysis of NVH problems.

\section{Smoothed finite-element formulation}

Consider a structural domain $\Omega_{s}$ coupled with a fluid domain $\Omega_{f}$, as shown in Fig. 1 . The fluid is enclosed by a flexible structure $\Omega_{s}$, together with a rigid boundary $\Gamma_{b}$ and an interface boundary $\Gamma_{a}$.

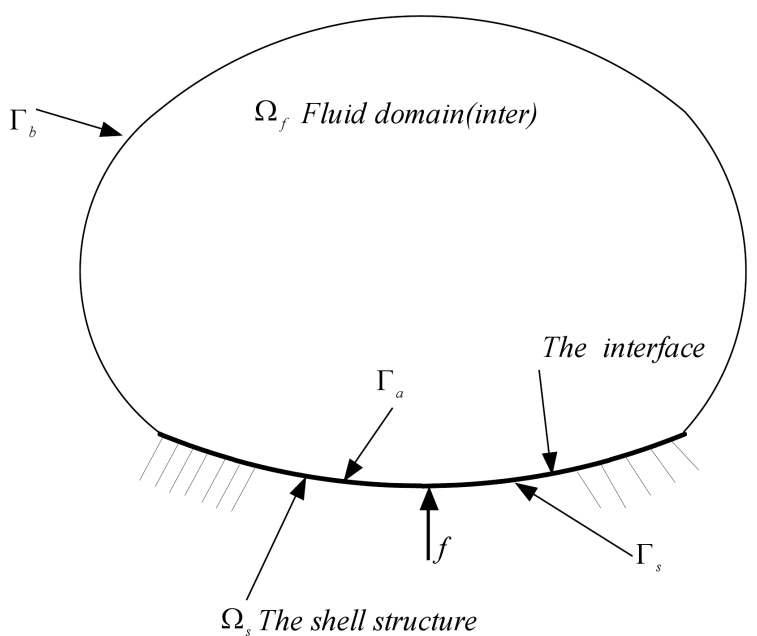

Fig. 1. Shell structure of domain $\Omega_{s}$ coupled with acoustic fluid of domain $\Omega_{f}$.

\subsection{Formulations for the shell}

In this section, the basic equations of shell theory are presented. Based on the first-order, sheardeformation theory, the displacements can be expressed as follows:

$$
\begin{aligned}
u(x, y, z) & =u_{0}(x, y)+z \theta_{x}(x, y) \\
v(x, y, z) & =v_{0}(x, y)+z \theta_{y}(x, y) \\
w(x, y, z) & =w_{0}(x, y)
\end{aligned}
$$

where $u_{0}, v_{0}$, and $w_{0}$ are the displacements of the midplane of the shell in the $x, y$, and $z$ directions; $\theta_{x}$ and $\theta_{y}$ are the rotations of the mid-plane around the $x$-axis and $y$-axis, respectively, as shown in Fig. 2.

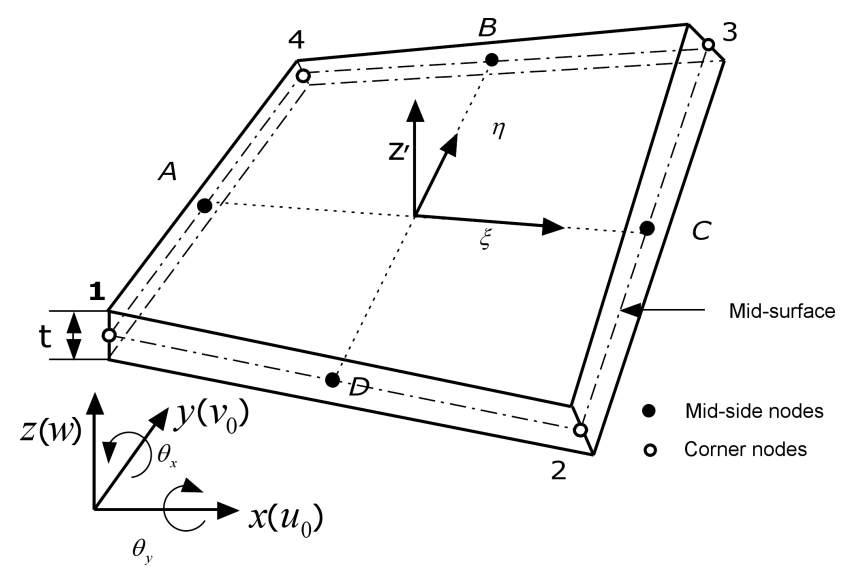

Fig. 2. Quadrilateral shell element. 
The strain vector $\varepsilon$ can be written in terms of the mid-plane deformations, which gives:

$$
\begin{aligned}
\varepsilon & =\left\{\varepsilon_{x x}, \varepsilon_{y y}, \gamma_{x y}, \gamma_{x z}, \gamma_{y z}\right\}^{\mathrm{T}} \\
& =\left\{\begin{array}{c}
\boldsymbol{\varepsilon}_{\mathbf{m}} \\
\mathbf{0}
\end{array}\right\}+\left\{\begin{array}{c}
z \varepsilon_{\mathbf{b}} \\
\mathbf{0}
\end{array}\right\}+\left\{\begin{array}{c}
\mathbf{0} \\
\boldsymbol{\varepsilon}_{\mathbf{s}}
\end{array}\right\},
\end{aligned}
$$

where $\boldsymbol{\varepsilon}_{m}$ is the membrane strain, $\boldsymbol{\varepsilon}_{b}$ is the bending strain, and $\varepsilon_{s}$ is the shear strain.

They can be given by:

$$
\begin{gathered}
\boldsymbol{\varepsilon}_{m}=\left[\begin{array}{c}
\frac{\partial u_{0}}{\partial x} \\
\frac{\partial v_{0}}{\partial y} \\
\frac{\partial u_{0}}{\partial y}+\frac{\partial v_{0}}{\partial x}
\end{array}\right], \quad \boldsymbol{\varepsilon}_{b}=\left[\begin{array}{c}
\frac{\partial \theta_{x}}{\partial x} \\
\frac{\partial \theta_{y}}{\partial y} \\
\frac{\partial \theta_{x}}{\partial y}+\frac{\partial \theta_{y}}{\partial x}
\end{array}\right], \\
\mathcal{\varepsilon}_{s}=\left[\begin{array}{c}
\frac{\partial w_{0}}{\partial x}+\theta_{x} \\
\frac{\partial w_{0}}{\partial y}+\theta_{y}
\end{array}\right] .
\end{gathered}
$$

Applying the principle of virtual work (PVW), the weak form of the dynamic variation equation can then be written as:

$$
\begin{aligned}
\int_{\Omega} \delta \boldsymbol{\varepsilon}_{m}^{\mathrm{T}} \mathbf{D}_{m} \boldsymbol{\varepsilon}_{m} \mathrm{~d} \Omega & +\int_{\Omega} \delta \bar{\varepsilon}_{b}^{\mathrm{T}} \mathbf{D}_{b} \boldsymbol{\varepsilon}_{b} \mathrm{~d} \Omega \\
& +\int_{\Omega} \delta \boldsymbol{\varepsilon}_{s}^{\mathrm{T}} \mathbf{D}_{s} \boldsymbol{\varepsilon}_{s} \mathrm{~d} \Omega+\int_{\Omega} \delta \mathbf{u}^{\mathrm{T}} \mathbf{m} \ddot{\mathbf{u}} \mathrm{d} \Omega \\
& -\int_{\Omega} \delta \mathbf{u}^{\mathrm{T}} f \mathrm{~d} \Omega=0
\end{aligned}
$$

in which, $f$ is the distributed load applied on the shell. $\mathbf{m}=\operatorname{diag}\left\{\mathbf{m}_{1} \mathbf{m}_{2} \mathbf{m}_{3} \mathbf{m}_{4}\right\}$ is the matrix containing the mass density $\rho_{s}$ and thickness $l$ with the form of

$$
\begin{aligned}
\mathbf{m}_{I} & =\operatorname{diag}\left\{\rho_{s} l \quad \rho_{s} l \quad \rho_{s} l \quad \rho_{s} l^{3} / 12 \quad \rho_{s} l^{3} / 12 \quad 0\right\}, \\
I & =1,2,3,4 .
\end{aligned}
$$

The field variable $\mathbf{u}$, the membrane stiffness constitutive coefficients $\mathbf{D}_{m}$, the bending stiffness constitutive coefficients $\mathbf{D}_{b}$, and the shear stiffness constitutive coefficients $\mathbf{D}_{s}$ can be expressed as:

$$
\begin{aligned}
\mathbf{u}(\mathbf{x}) & =\sum_{I=1}^{N p} \mathbf{N}_{I}(\mathbf{x}) \mathbf{d}_{I}, & \delta \mathbf{u}(\mathbf{x}) & =\sum_{I=1}^{N p} \mathbf{N}_{I}(\mathbf{x}) \delta \mathbf{d}_{I}, \\
\boldsymbol{\varepsilon}_{m} & =\sum_{I=1}^{N p} \mathbf{B}_{m I} \mathbf{d}_{I}, & \boldsymbol{\varepsilon}_{b} & =\sum_{I=1}^{N p} \mathbf{B}_{b I} \mathbf{d}_{I}, \\
\boldsymbol{\varepsilon}_{s} & =\sum_{I=1}^{N p} \mathbf{B}_{s I} \mathbf{d}_{I}, & \ddot{\mathbf{u}}(\mathbf{x}) & =\sum_{I=1}^{N p} \mathbf{N}_{I}(\mathbf{x}) \ddot{\mathbf{d}}_{I},
\end{aligned}
$$

$$
\begin{aligned}
& \mathbf{D}_{m}=\frac{E l}{(1-\nu)^{2}}\left[\begin{array}{ccc}
1 & \nu & 0 \\
\nu & 1 & 0 \\
0 & 0 & \frac{1-\nu}{2}
\end{array}\right], \\
& \mathbf{D}_{b}=\frac{E l^{3}}{12\left(1-v^{2}\right)}\left[\begin{array}{ccc}
1 & v & 0 \\
v & 1 & 0 \\
0 & 0 & \frac{1-v}{2}
\end{array}\right], \\
& \mathbf{D}_{s}=\frac{E l k}{2(1+v)}\left[\begin{array}{ll}
1 & 0 \\
0 & 1
\end{array}\right],
\end{aligned}
$$

where $E$ is Young's modulus, $v$ is Poisson's ratio, and $k$ is the shear correction factor, $N p$ is the number of nodes in the element, $\mathbf{d}_{I}=\left[u_{I}, v_{I}, w_{I}, \theta_{x I}, \theta_{y I}\right]^{\mathrm{T}}$ is the generalized nodal displacement at node $I$, and $\mathbf{N}_{I}(\mathbf{x})$ is the shape functions defined as:

$\mathbf{N}_{I}(\mathbf{x})=\operatorname{diag}\left(N_{I}(\mathbf{x}), N_{I}(\mathbf{x}), N_{I}(\mathbf{x}), N_{I}(\mathbf{x}), N_{I}(\mathbf{x})\right)$,

where $N_{I}(\mathbf{x})$ is the shape function associated to node $I$ and $\mathbf{B}_{m I}, \mathbf{B}_{b I}$ and $\mathbf{B}_{s I}$ are the strain matrix defined as:

$$
\begin{aligned}
\mathbf{B}_{m I} & =\left(\begin{array}{ccccc}
N_{I, x} & 0 & 0 & 0 & 0 \\
0 & N_{I, y} & 0 & 0 & 0 \\
N_{I, y} & N_{I, x} & 0 & 0 & 0
\end{array}\right), \\
\mathbf{B}_{b I} & =\left(\begin{array}{ccccc}
0 & 0 & 0 & N_{I, x} & 0 \\
0 & 0 & 0 & 0 & N_{I, y} \\
0 & 0 & 0 & N_{I, y} & N_{I, x}
\end{array}\right), \\
\mathbf{B}_{s I} & =\left[\begin{array}{lllll}
0 & 0 & N_{I, x} & N_{I} & 0 \\
0 & 0 & N_{I, y} & 0 & N_{I}
\end{array}\right] .
\end{aligned}
$$

Substituting Eq. (6) into Eq. (4) yields:

$$
\mathbf{K u}-\mathbf{M} \ddot{\mathbf{u}}=\mathbf{F}_{s},
$$

where the smoothed stiffness matrix $\overline{\mathbf{K}}$ can be expressed as:

$$
\begin{aligned}
\mathbf{K} & =\mathbf{K}^{m}+\mathbf{K}^{b}+\mathbf{K}^{s} \\
& =\int_{\Omega} \mathbf{B}_{m}^{T} \mathbf{D}_{m} \mathbf{B}_{m}+\int_{\Omega} \mathbf{B}_{b}^{T} \mathbf{D}_{b} \mathbf{B}_{b}+\int_{\Omega} \mathbf{B}_{s}^{T} \mathbf{D}_{s} \mathbf{B}_{s} .
\end{aligned}
$$

Mass matrix $\mathbf{M}$ can be written as:

$$
\mathbf{M}=\int_{\Omega} \mathbf{N}^{\mathrm{T}} \mathbf{m} \mathbf{N} \mathrm{d} \Omega
$$

The force vector $\mathbf{F}_{s}$ is:

$$
\mathbf{F}_{s}=\int_{\Omega} \mathbf{N}^{\mathrm{T}} f \mathrm{~d} \Omega
$$


Considering harmonic forced excitations one can substitute $\mathbf{u}=\mathbf{U} e^{j \omega t}$ into Eq. (10), where $j=\sqrt{-1}$ and $t$ is the time. The forced frequency response analysis equation can be written as:

$$
\left(\mathbf{K}-\omega^{2} \mathbf{M}\right) \mathbf{U}=\mathbf{F}_{s} .
$$

It is obvious that the smoothed membrane and bending strain matrix integration can be solved more easily and efficiently.

\subsection{Cell-based strain smoothing operation for the shell element}

In this sub-section, the cell-based smoothing technique for the shell element is formulated. The shell domain is discretized into $N_{e}$ quadrilateral elements with $N_{d}$ nodes in the standard FEM. Each element domain $\Omega$ is divided into $S C$ smoothing cells, such that $\Omega_{1} \cup \Omega_{2} \cup \ldots \Omega_{S C}=\Omega$ and $\Omega_{1} \cap \Omega_{2} \cap \ldots \Omega_{S C}=\varnothing$. These smoothing cells are shown in Fig. 3 . Figure 3 illustrates different smoothing cells for $\mathrm{SC}=1,2,3$, and 4 corresponding to 1st smoothing cells, 2nd smoothing cells, 3rd smoothing cells, and 4th smoothing cells methods. The membrane and bending are smoothed over each smoothing cells. The values of the shape functions are indicated at the corner nodes in Fig. 3 in the format $\left(N_{1}, N_{2}, N_{3}, N_{4}\right)$. The values of the shape functions at the integration nodes are determined based on the lin- ear interpolation of shape functions along boundaries of the element or the smoothing cells.

Applying the smoothing operation on the bending and membrane strain of the shell, the compatible bending and membrane strain in the $C$-th smoothing domain can be calculated using:

$$
\begin{gathered}
\bar{\varepsilon}_{b c}=\int_{\Omega_{c}} \varepsilon_{b}(\mathbf{x}) \mathrm{H}(\mathbf{x}) \mathrm{d} \Omega, \\
\bar{\varepsilon}_{m c}(\mathbf{x})=\int_{\Omega_{C}} \varepsilon_{m}(\mathbf{x}) \mathrm{H}(\mathbf{x}) \mathrm{d} \Omega,
\end{gathered}
$$

where $\mathrm{H}(x)$ is a given smoothing function that satisfies at least unity property

$$
\int_{\Omega_{C}} H(\mathbf{x})=1 .
$$

In this paper, we use the constant smoothing function defined as

$$
\mathrm{H}(\mathbf{x})=\left\{\begin{array}{cc}
1 / A_{C} & \mathbf{x} \in \Omega_{C}, \\
0 & \mathbf{x} \notin \Omega_{C},
\end{array}\right.
$$

where $A_{C}=\int_{\Omega_{C}} \mathrm{~d} \Omega$ is the area of $C$-th smoothing domain.
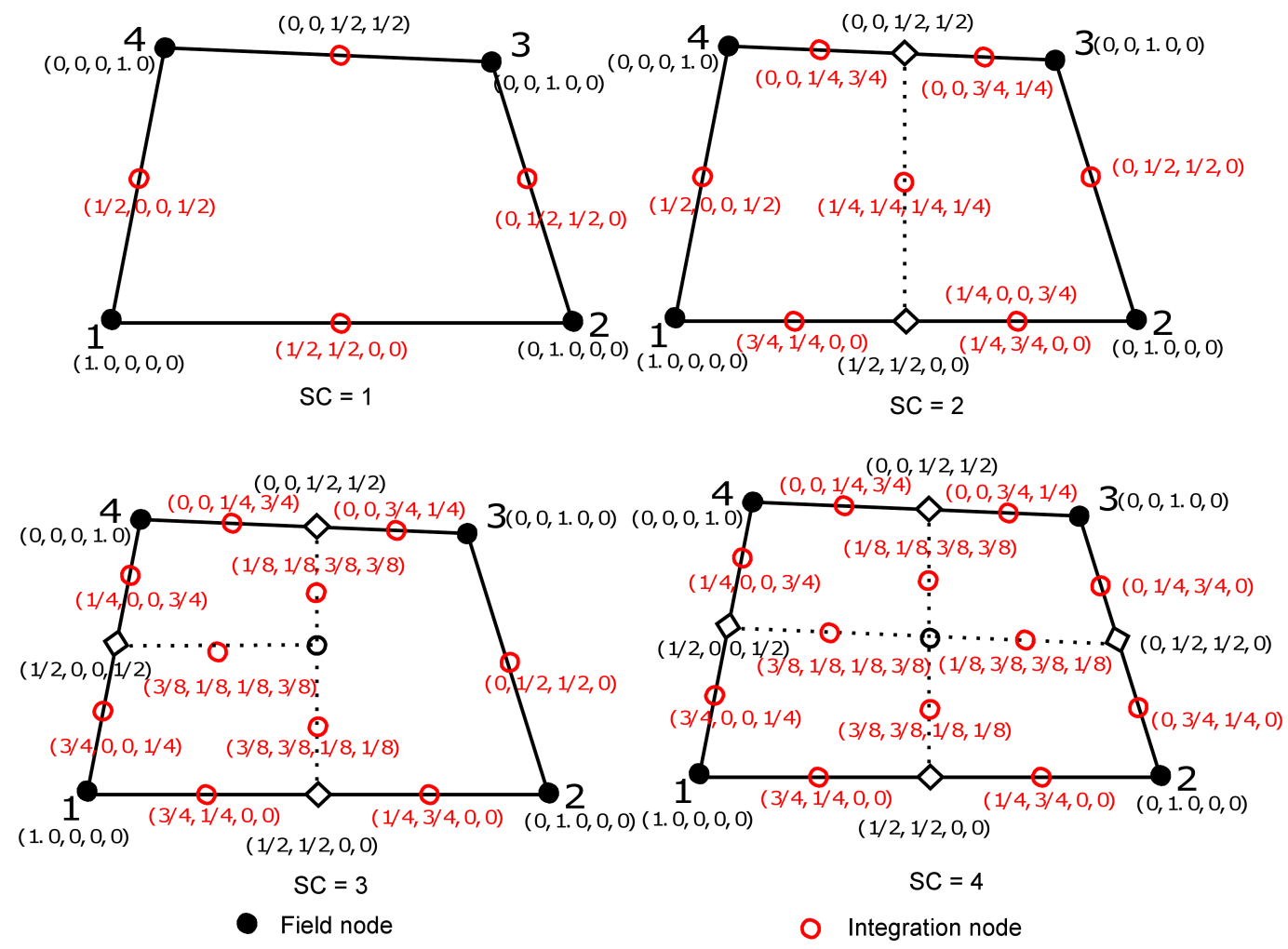

Fig. 3. Division of a quadrilateral element into smoothing cells (SC) and the value of the shape function along the boundaries of cells. 
Substituting Eqs. (6), (8) and (17) into Eq. (15) and applying a divergence theorem, one can get the smoothed membrane strain:

$$
\begin{aligned}
\bar{\varepsilon}_{m C}(\mathbf{x}) & =\frac{1}{A_{C}} \int_{\Omega_{C}} \nabla s \mathbf{u}^{h}(\mathbf{x}) \mathrm{d} \Omega_{C} \\
& =\frac{1}{A_{C}} \int_{\Gamma_{C}} \mathbf{n}_{C} \mathbf{u}^{h}(\mathbf{x}) \mathrm{d} \Gamma_{C}=\sum_{I=1}^{N p} \overline{\mathbf{B}}_{m I}^{C}(\mathbf{x}) \mathbf{d}_{I},
\end{aligned}
$$

where $\Gamma_{c}$ is the boundary of the smoothed cell, $\mathbf{n}_{C}$ is the outward normal vector matrix on the boundary $\Gamma_{c}$, and $\nabla s$ is a differential operator matrix defined as

$$
\nabla s=\left[\begin{array}{ccccc}
\frac{\partial}{\partial x} & 0 & 0 & 0 & 0 \\
0 & \frac{\partial}{\partial y} & 0 & 0 & 0 \\
\frac{\partial}{\partial y} & \frac{\partial}{\partial x} & 0 & 0 & 0
\end{array}\right]
$$

and the $\overline{\mathbf{B}}_{m I}^{C}(\mathbf{x})$ is the smoothed membrane strain matrix defined as:

$\overline{\mathbf{B}}_{m I}^{c}(\mathbf{x})=\frac{1}{A_{C}} \int_{\Gamma_{C}}\left(\begin{array}{ccccc}N_{I} n_{x} & 0 & 0 & 0 & 0 \\ 0 & N_{I} n_{y} & 0 & 0 & 0 \\ N_{I} n_{y} & N_{I} n_{x} & 0 & 0 & 0\end{array}\right) \mathrm{d} \Gamma$.

Similarly, the smoothed bending strain $\bar{\varepsilon}_{b C}^{h}$ over the domain can be expressed as:

$$
\overline{\boldsymbol{\varepsilon}}_{b C}(\mathbf{x})=\sum_{I=1}^{N p} \overline{\mathbf{B}}_{b I}^{C}(\mathbf{x}) \mathbf{d}_{I},
$$

where $\overline{\mathbf{B}}_{b I}^{c}(\mathbf{x})$ can be written as:

$$
\overline{\mathbf{B}}_{b I}^{C}(\mathbf{x})=\frac{1}{A_{C}} \int_{\Gamma_{C}}\left(\begin{array}{ccccc}
0 & 0 & 0 & N_{I} n_{x} & 0 \\
0 & 0 & 0 & 0 & N_{I} n_{y} \\
0 & 0 & 0 & N_{I} n_{y} & N_{I} n_{x}
\end{array}\right) \mathrm{d} \Gamma .
$$

The smoothed membrane and bending strains lead to high flexibility such as arbitrary polygonal elements, and a slight reduction in computational cost.

Simultaneously, we use independent interpolation fields in the natural coordinate system for the approximation of the shear strains (FREDRIK, 2001), and the $2 \times 2$ Gauss quadrature is still utilized, like in (FREDRIK, 2001).

Finally, the smoothed stiffness matrix $\overline{\mathbf{K}}$ can be expressed as:

$$
\begin{aligned}
\overline{\mathbf{K}}= & \overline{\mathbf{K}}^{m}+\overline{\mathbf{K}}^{b}+\mathbf{K}^{s}=\sum_{C=1}^{S C}\left(\overline{\mathbf{B}}_{m I}^{C}\right)^{T} \mathbf{D}_{m} \overline{\mathbf{B}}_{m I}^{C} A_{C} \\
& +\sum_{C=1}^{S C}\left(\overline{\mathbf{B}}_{b I}^{C}\right)^{T} \mathbf{D}_{b} \overline{\mathbf{B}}_{b I}^{C} A_{C} \\
& +\sum_{i=1}^{2} \sum_{j=1}^{2} w_{i} w_{j}\left(\mathbf{B}_{s}\right)^{T} \mathbf{D}_{s} \mathbf{B}_{s}|\mathbf{J}| \mathrm{d} \xi \mathrm{d} \eta
\end{aligned}
$$

According to Eq. (2), after the smoothing operation was implemented on the smoothed domains of the shell, the smoothed strains vector, $\bar{\varepsilon}$, can be written as:

$$
\bar{\varepsilon}=\left\{\begin{array}{c}
\bar{\varepsilon}_{m} \\
\mathbf{0}
\end{array}\right\}+\left\{\begin{array}{c}
z \bar{\varepsilon}_{b} \\
\mathbf{0}
\end{array}\right\}+\left\{\begin{array}{c}
\mathbf{0} \\
\varepsilon_{\mathbf{s}}
\end{array}\right\} .
$$

\section{Formulations of the fluid sub-domain}

In the (acoustic) fluid sub-domain, the governing equation for the linear acoustic problems known as the Helmholtz equation is given by

$$
\nabla^{2} p+k^{2} p=0 \quad \text { with } \quad k=\omega / c,
$$

where $\nabla^{2}$ denotes the Laplace operator, and the wave number $k$ is determined by the ratio between the angular frequency $\omega$ and the speed of sound $c, p(x, t)=$ $p(x) \cdot e^{j \omega t}$. The acoustic boundary conditions include the rigid wall condition can be expressed as follows:

$$
\frac{\partial p}{\partial n}=0 \quad \text { on } \quad \Gamma_{f},
$$

where the $n$ is the exterior unit normal vector of the acoustic fluid. At the interface of the fluid and the structure, the momentum balance require that

$$
\frac{\partial p}{\partial n}=\rho \omega^{2} u_{n} \quad \text { on } \quad \Omega_{s f},
$$

where $\rho$ is the density of medium and the normal displacement component of the acoustic fluid at the interface, and the symbol $\Omega_{s f}$ denotes interface between the acoustic fluid and structure. The subscript "sf" stands for the interface.

The boundary integral equation for acoustic problems can be used for the interior and exterior problems. The weak form of the Helmholtz equation can be obtained using a weighted residuals approach with the following fundamental solution as the weight function:

$$
G=e^{-i k r} / r,
$$

where $r=|\bar{Q}-\bar{P}|, P$ is a collocation point and $Q$ is a source point.

Using Green's second identity theorem, the Helmholtz equation in Eq. (25) can be transformed into a boundary integral equation, which relates the volume integral over the domain $V$ to the surface integral on the boundary $S$, and, using the standard boundary element procedure, we can get the boundary integral equation:

$$
C(P) p(P)=\int_{S}\left(\frac{\partial p}{\partial n} G-p \frac{\partial G}{\partial n}\right) \mathrm{d} S,
$$

where $n$ is the outward normal at point $\mathrm{Q} ; C(P)$ is a coefficient, which depends on the position of the point $P$. 
The value of $C(P)$ is calculated by a more general expression defined as:

$$
C(P)=\left\{\begin{array}{cc}
1 & P \in V, \\
4 \pi \cdot \operatorname{sign}+\int_{S} \frac{\partial G_{0}}{\partial n} \mathrm{~d} S & P \in S, \\
0 & P \notin S, V,
\end{array}\right.
$$

where $G_{0}=1 / r$. By taking into account the Neumann boundary condition given by Eq. (27) about the relation between the normal derivative of the sound pressure and velocity, then Eq. (29) becomes

$$
C(P) p(P)=\int_{S}\left(\rho \omega^{2} u_{n} G+p \frac{\partial G}{\partial n}\right) \mathrm{d} S,
$$

$p$ and $u_{n}$ will vary along the boundary and can be approximated by a simple function; hence they can be expressed as a function of their nodal values.

$$
\begin{gathered}
p=\sum_{j=1}^{m} \mathbf{N}_{j} p_{j}=\mathbf{N}_{f} \mathbf{p}, \\
u_{n}=\sum_{j=1}^{m} \mathbf{N}_{j} u_{n j}=\mathbf{N}_{f} \mathbf{u}_{n},
\end{gathered}
$$

where $p_{j}$ and $u_{n j}$ are the nodal values of acoustic pressure and particle normal velocity at node $j$, respectively, $m$ is the number of nodes on each surface element and $\mathbf{N}_{f}$ are the shape functions. Assume that the boundary of the domain is discretized into $N$ isoparameteric, quadrilateral elements with $M$ nodes.

We then place the point $P$ at each of nodal points on the boundary successively which is known as "collocation". For each collocation point $P$, we substitute Eq. (32) into Eq. (31) and perform the integration over the entire surface, which is actually done on an element-by-element basis. A set of simultaneous linear equation can be obtained when point $P$ is chosen to be all the nodal points on the boundary and the discretized form can be expressed as (NEHETE et al., 2015; FREDRIK, 2001):

$$
C_{i} \delta_{i j} p_{i}=\sum_{j=1}^{N} \widehat{G}_{i j} \frac{\partial p}{\partial n}-\sum_{j=1}^{N} \widehat{H}_{i j} p_{i} .
$$

Combining the constant term $C$ with $\widehat{\mathbf{H}}$ matrix, Eq. (33) can be expressed in matrix form as:

$$
\mathbf{H P}=\mathbf{G} \frac{\partial p}{\partial n}
$$

where

$$
\begin{gathered}
\mathbf{H}=\sum_{j=1}^{N} C_{i} \delta_{i j}+\widehat{H}_{i j}, \quad \delta_{i j}= \begin{cases}1 & i=j, \\
0 & i \neq j,\end{cases} \\
\mathbf{G}=\sum_{j=1}^{N} G_{i j}, \quad \widehat{H}_{i j}=\int_{S_{i}} \frac{\partial p}{\partial n} \mathrm{~d} s, \quad G_{i j}=\int_{S_{i}} p \mathrm{~d} s
\end{gathered}
$$

and $S_{j}$ is the surface of element $j$ on the boundary. Combining Eqs. (26), (27) and (34), we can get:

$$
\mathbf{H P}=-\rho_{f} \omega^{2} \mathbf{G} \mathbf{u}_{n},
$$

where $\rho_{f}$ is the fluid density.

\section{Coupling of SFEM/BEM for structure-acoustic coupled analysis}

The general formulation of fluid-structure interaction presented in this section is based on the formulations for the shell and for acoustic fluid. During the interaction, the fluid particle and the structure move together in the normal direction of the boundary, and the interface boundary condition can be written as:

$$
u_{s} \mathbf{n}=u_{f} \mathbf{n},
$$

where $\mathbf{n}$ is the normal vector along the fluid boundary, $u_{s}$ is the displacement of the shell at the interface, and $u_{f}$ is the displacement of the fluid contacting the shell. At the interface, the continuity in pressure can be expressed as follows:

$$
\left.p_{s}\right|_{n}=-p_{f},
$$

where the subscript " $f$ " stands for boundary element nodes of the fluid and subscript " $s$ " stands for shell element nodes of the structure at the fluid-structure interface; $\left.p_{s}\right|_{n}$ stands for the normal pressure of shell element nodes at the interface; $p_{f}$ stands for the pressure of the fluid at the interface. Note that the normal on the interface on the shell $\mathbf{n}_{s}$ is opposite the normal on the fluid $\mathbf{n}_{f}$, that is:

$$
\mathbf{n}=\mathbf{n}_{s}=-\mathbf{n}_{f} .
$$

Under the equilibrium conditions (27), the motion Eq. (14) can be given in the frequency domain:

$$
\left(\overline{\mathbf{K}}-\omega^{2} \mathbf{M}\right) \mathbf{U}=\mathbf{F}_{s}+\mathbf{F}_{f},
$$

where $\mathbf{F}_{f}$ is the fluid force load on the structure boundary $\Omega_{s f}$ and can be expressed as:

$$
\mathbf{F}_{f}=-\int_{\Omega_{s f}} \mathbf{N}^{\mathrm{T}} p_{f} \mathbf{n} \mathrm{d} S .
$$

By introducing the spatial coupling matrix $\mathbf{L}$ :

$$
\mathbf{L}=\sum_{e} \int_{s_{e}} \mathbf{N}^{\mathrm{T}} \mathbf{n} \mathbf{N}_{B} \mathrm{~d} s,
$$

where $\mathbf{N}_{B}$ is the interpolation function for a four-node quadrilateral BEM element. Then, Eq. (40) can be expressed as:

$$
\left(\overline{\mathbf{K}}-\omega^{2} \mathbf{M}\right) \mathbf{U}+\mathbf{L} \mathbf{P}_{f}=\mathbf{F}_{s}
$$


Considering the boundary conditions of the fluid domain, the BEM model is divided into two parts, $a$ and $b$, as shown in Fig. 4.

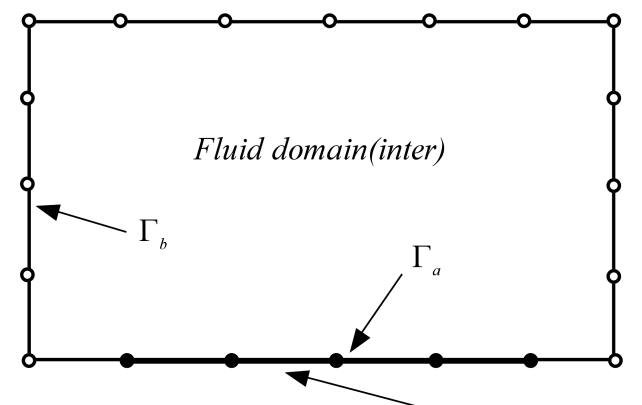

The shell elements on interface

- nodes of interface o nodes of non-interface

Fig. 4. The SFEM shells coupled with boundary elements.

The boundary node pressure values $p$ are sorted into two groups, $p_{a}$ and $p_{b}$, depending on the boundary upon which they act. The node normal displacement $\mathbf{u}_{n}$ is divided into two parts, $\mathbf{u}_{a}$ and $\mathbf{u}_{b}$. Then, Eq. (33) can be rewritten as:

$$
\left[\begin{array}{ll}
\mathbf{H}_{11} & \mathbf{H}_{12} \\
\mathbf{H}_{21} & \mathbf{H}_{22}
\end{array}\right]\left[\begin{array}{l}
\mathbf{p}_{a} \\
\mathbf{p}_{b}
\end{array}\right]=-\rho_{f} \omega^{2}\left[\begin{array}{ll}
\mathbf{G}_{11} & \mathbf{G}_{12} \\
\mathbf{G}_{21} & \mathbf{G}_{22}
\end{array}\right]\left[\begin{array}{l}
\mathbf{u}_{a} \\
\mathbf{u}_{b}
\end{array}\right]
$$

and we can get:

$$
\begin{aligned}
& \frac{\partial p}{\partial n}=\rho_{f} \omega^{2} u_{n}=\rho_{f} \omega^{2} u_{a} \mathbf{n}_{a}, \\
& \frac{\partial p}{\partial n}=\rho_{f} \omega^{2} u_{n}=\rho_{f} \omega^{2} u_{b} \mathbf{n}_{b},
\end{aligned}
$$

where $\mathbf{n}_{a}$ and $\mathbf{n}_{b}$ are the outward surface normal vectors on the fluid boundaries $\Gamma_{a}$ and $\Gamma_{b}$, respectively.

Combining the structural SFEM model with the acoustic BEM model yields the coupled SFEM/BEM model:

$$
\left[\begin{array}{ccc}
\overline{\mathbf{K}}-\omega^{2} \mathbf{M} & \mathbf{L} & 0 \\
\rho_{f} \omega^{2} \mathbf{G}_{11} \mathbf{n}_{a} & \mathbf{H}_{11} & \mathbf{H}_{12} \\
\rho_{f} \omega^{2} \mathbf{G}_{21} \mathbf{n}_{a} & \mathbf{H}_{12} & \mathbf{H}_{22}
\end{array}\right]\left[\begin{array}{c}
\mathbf{U} \\
\mathbf{p}_{a} \\
\mathbf{p}_{b}
\end{array}\right]=\left[\begin{array}{c}
\mathbf{F} \\
\mathbf{F}_{a} \\
\mathbf{F}_{b}
\end{array}\right],
$$

where

$$
\mathbf{F}_{a}=-\rho_{f} \omega^{2} \mathbf{G}_{12} \mathbf{n}_{b} \mathbf{u}_{b}, \quad \mathbf{F}_{b}=-\rho_{f} \omega^{2} \mathbf{G}_{22} \mathbf{n}_{b} \mathbf{u}_{b}
$$

\section{Application examples}

In this section, there are two examples of fluidstructure interaction problems used to verify the present SFEM-BEM formulation. We tested the computer program developed based on the proposed formulations. SYSNOISE software was used as a reference in order to validate the numerical model that couples the finite-element method (FEM) with the boundary element method (BEM). Some conclusions were obtained by comparing the reference data with the numerical results.

\subsection{Box structural-acoustic problem}

Considering a box cavity surrounded by five rigid surfaces and one elastic surface. The dimensions of this box cavity are $0.4 \times 0.4 \times 0.4 \mathrm{~m}$. The box cavity is filled with a fluid which is attached to a flexible flat shell. The fluid is air with material properties of $\rho_{s}=1.225 \mathrm{~kg} / \mathrm{m}^{3}, c=343 \mathrm{~m} / \mathrm{s}$. The flexible shell made of steel $\left(\rho_{s}=7800 \mathrm{~kg} / \mathrm{m}^{3}, v=0.3\right.$, and $E=30 \mathrm{GPa}$ ) is on the bottom of the box cavity. The thickness of this flexible shell is $0.001 \mathrm{~m}$, and exhibits simply supported boundary conditions, excited by a point time harmonic force loading at the center of the panel. The box cavity walls, including the rigid wall and the flexible wall, are modeled with boundary elements. The fluid-structure interaction model, as described in (WANG et al., 2015) is shown in Fig. 5. In this numerical example, the structure and the fluid domain boundary are meshed with quadrilateral elements, and the damping is neglected. To study the performance of the SFEM for the two-dimensional (2D) structural flat shell, the natural eigenfrequencies analyses for the 2D flexible shell were investigated first. The shell was discretized into 81 nodes and 64 quadrilateral elements with an average mesh size of $0.05 \mathrm{~m}$. Table 1 lists the first fifteen non-rigid eigenfrequencies of the flat shell obtained from SFEM. To compare with the FEM, the flexible shell modeled with the shell element using the same nodes and elements was computed by the FEM code, and the FEM numerical solutions of 15 non-rigid eigenfrequencies are listed in Table 1. As the FEM/BEM can provide appropriate result when the mesh size is sufficiently small, the reference result obtained using FEM in SYSNOISE with a very fine quadrilateral mesh (1681 nodes and 1600 elements) is also employed to make a comparison, and the result is also provided in the table. Table 1 shows that 1 ) the eigenfrequencies computed using FEM were all larger than the reference solution and 2) the deviation of eigenfrequencies between the FEM solution and

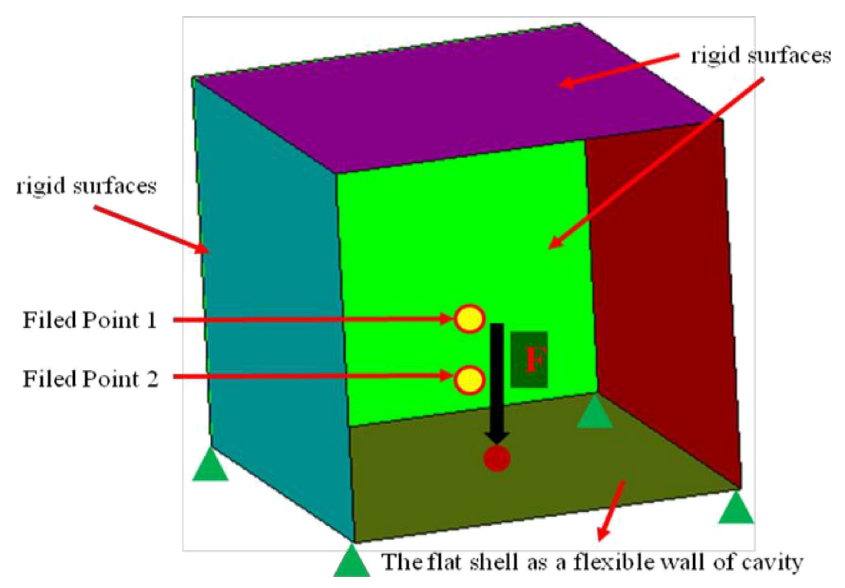

Fig. 5. Box cavity with one flexible wall. 
Table 1. Eigenfrequencies of flat shell obtained using SFEM and FEM.

\begin{tabular}{|c|c|c|c|c|c|}
\hline Mode number & SFEM freq. [Hz] & Error [\%] & FEM freq. [Hz] & Error [\%] & Reference freq. [Hz] \\
\hline 1 & 59.3433 & 4.96 & 62.2131 & 10.03 & 56.5394 \\
\hline 2 & 119.7178 & 3.10 & 126.7201 & 9.13 & 116.1183 \\
\hline 3 & 120.9435 & 3.43 & 126.7201 & 8.37 & 116.9321 \\
\hline 4 & 150.4299 & 3.14 & 157.9926 & 8.32 & 145.8554 \\
\hline 5 & 286.6578 & 5.74 & 294.4624 & 8.62 & 271.0963 \\
\hline 6 & 291.5923 & 3.50 & 304.2299 & 7.99 & 281.7274 \\
\hline 7 & 360.1008 & 5.27 & 380.8492 & 11.34 & 342.0635 \\
\hline 8 & 363.6481 & 5.85 & 380.8492 & 10.86 & 343.5464 \\
\hline 9 & 492.4435 & 5.39 & 522.1091 & 11.74 & 467.2582 \\
\hline 10 & 542.0775 & 4.65 & 561.488 & 8.40 & 517.9904 \\
\hline 11 & 539.0406 & 4.06 & 561.488 & 8.39 & 518.0246 \\
\hline 12 & 689.7948 & 9.97 & 748.5495 & 19.33 & 627.2757 \\
\hline 13 & 840.5132 & 8.46 & 873.8227 & 12.76 & 774.9457 \\
\hline 14 & 881.8973 & 13.4 & 910.3988 & 17.07 & 777.6849 \\
\hline 15 & 889.8116 & 13.87 & 923.2459 & 18.14 & 781.4614 \\
\hline
\end{tabular}

the reference became larger as the eigenfrequencies increased. However, the eigenfrequencies obtained from the SFEM were closer in magnitude to the reference than those from the FEM solutions.

To study the performance of the coupled SFEM/BEM for fluid-structure interaction analysis, the forced frequency response analysis for the coupled cavity is then investigated using present coupled SFEM/BEM and the coupled FEM/BEM. A unit load is applied at the center of flat shell, and the acoustic response is measured at the field points 1 and 2. These field points were located in the fluid domain, just above the centre of the vibrating panel at distances of $0.1 \mathrm{~m}$ and $0.05 \mathrm{~m}$, respectively. The frequencies ranging from 50 to $150 \mathrm{~Hz}$ are adopted to investigate these two formulations. The frequency responses obtained using coupled SFEM/BEM and coupled FEM/BEM are plotted in Figs. 6 and 7, respectively. Since there was no analytical result for this coupled system, the reference responses obtained using coupled FEM/BEM with very fine mesh(quadrilateral mesh of 1681 nodes for the flat shell and quadrilateral mesh of 6724 nodes for cavity wall) in SYSNOISE are also presented in these figures. Figures 6 and 7 show that the results obtained from the coupled SFEM/BEM are in good agreement with the reference results at these frequencies; while the deviations between the coupled FEM/BEM and the reference all increased as frequency increased.

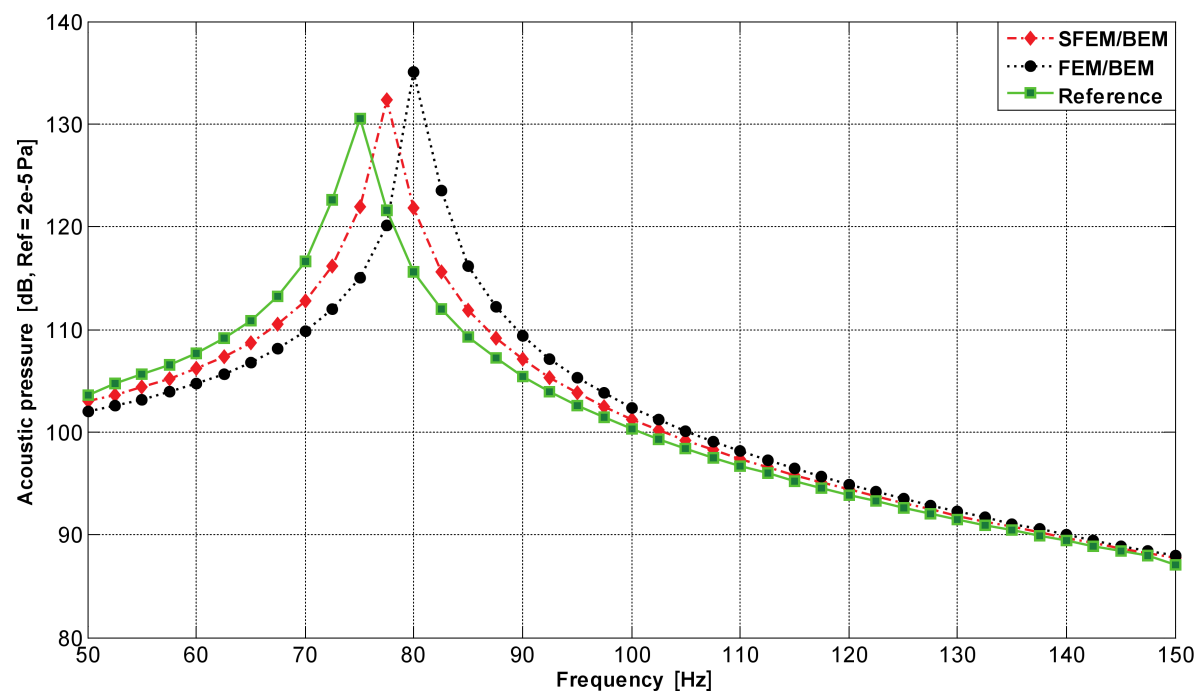

Fig. 6. The acoustic pressure frequency responses of field point 1. 


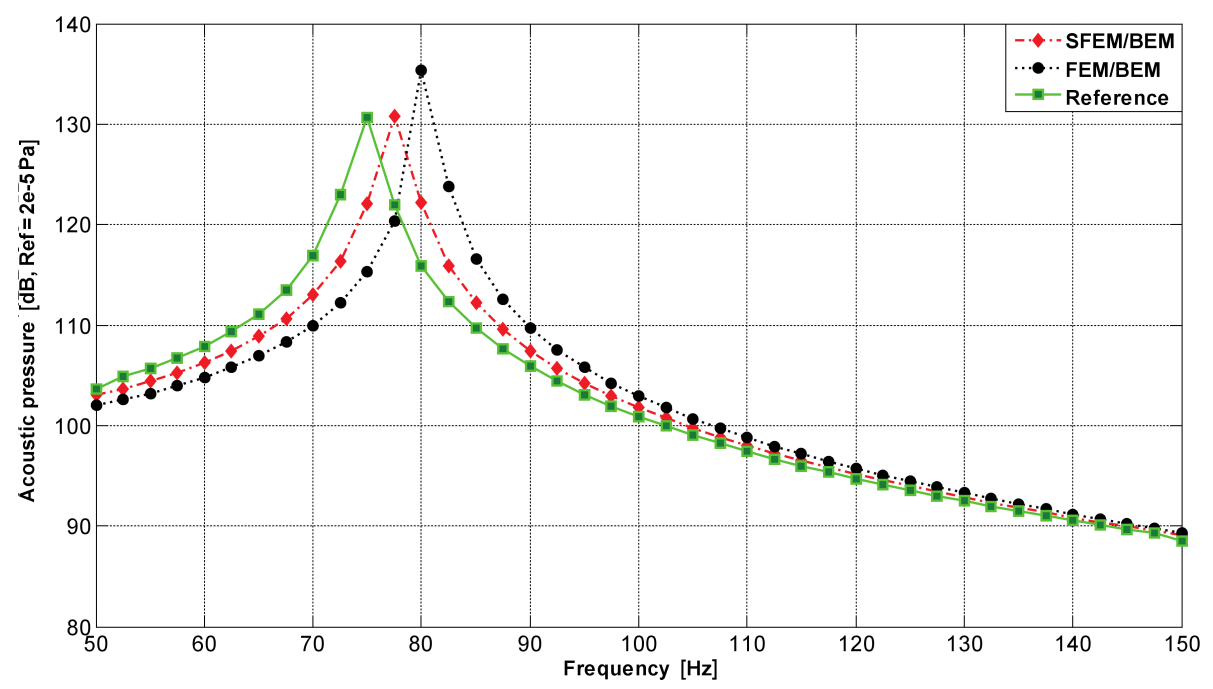

Fig. 7. The acoustic pressure frequency responses of field point 2.

\subsection{Automobile passenger compartment with flexible floor panel}

In many engineering situations, such as a vehicle passenger compartment, noise and vibration problems generated by fluid-structure interaction are often encountered. The interior noise level of such an enclosure is directly affected by the coupling between the flexible structure and an acoustic cavity. In this subsection, a simple model of coupled vehicle passenger compartment system, which is shown in Fig. 8, is used to investigate the performance of coupled SFEM/BEM and coupled FEM/BEM. Since the chassis is the major source of structural vibration and induces noise in the vehicle compartment, the coupling between the floor panel and the passenger compartment cavity is of great concern in reducing the noise level. The material of the shell and the acoustic fluid are the same as Subsec. 5.1. The floor panel was modeled as shell structure with all corners fixed, damping neglected, the vehicle passenger compartment was modeled with boundary elements, the floor panel was meshed with 60 quadrilateral elements, and the fluid domain boundary was meshed 236 quadrilateral elements.

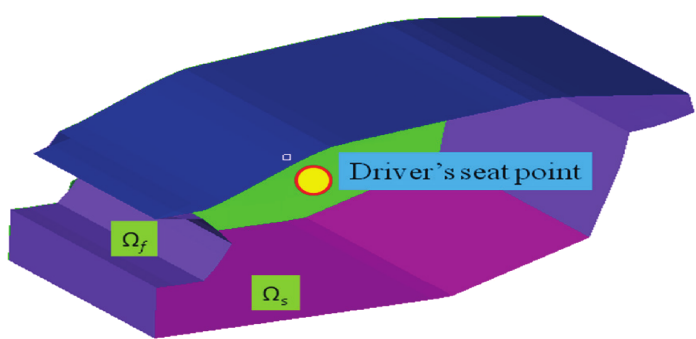

Fig. 8. Structural domain for the floor panel couples with the acoustical fluid domain.

Similar to the first example, the eigenfrequencies of the vehicle floor panel system were investigated using the SFEM, and the first 10 eigenfrequencies are listed in Table 2. For comparison, the results obtained from FEM with the same mesh are also provided in Table 2. In order to make a comparison, the reference results obtained using the coupled FEM in SYSNOISE with a fine mesh (quadrilateral mesh of 961nodes for the floor panel and boundary quadrilateral element of 8794 nodes for air cavity) is also employed and presented in Table 2. Table 2 shows that the errors of the eigenfrequencies of the floor panel using SFEM were smaller than the errors obtained from the FEM. These results validate that the cell-based strain-smoothing technique provides more accurate stiffness to the floor panel system, and therefore gives better results than FEM.

The forced frequency response for the coupled vehicle passenger compartment was also evaluated. A unit load was applied at the centre of the floor panel, and the responses were measured at the driver's seat point, in the vehicle passenger compartment of acoustic fluid. Frequencies ranging from $50 \mathrm{~Hz}$ to $150 \mathrm{~Hz}$, which are of great concern in developing a vehicle, were used to investigate the formulation of the coupled SFEM/BEM. The frequency responses of the driver's seat point using coupled SFEM/BEM and coupled FEM/FEM are plotted in Fig. 9, respectively. For comparison, the reference results obtained from coupled FEM/BEM in SYSNOISE with a fine mesh (quadrilateral mesh of 961 nodes for the floor panel and boundary quadrilateral element of 8794 nodes for air cavity) are also presented in these figures. As depicted in these figures, the results obtained from the coupled SFEM/BEM are in good agreement with the reference results at these frequencies, while there were significant deviations between the results obtained from coupled FEM/BEM and the reference results. The figure shows that the new method, i.e., coupled SFEM/BEM, can provide more accurate results than the corresponding FEM/BEM approach when used to solve industrial problems. 
Table 2. Eigenfrequencies of floor panel obtained using SFEM and FEM.

\begin{tabular}{|c|c|c|c|c|c|}
\hline Mode number & SFEM freq. $[\mathrm{Hz}]$ & Error [\%] & FEM freq. $[\mathrm{Hz}]$ & Error [\%] & Reference freq. [Hz] \\
\hline 1 & 61.8096 & 1.147 & 64.5890 & 5.69 & 61.1089 \\
\hline 2 & 145.0382 & 1.16 & 149.9892 & 4.61 & 143.3753 \\
\hline 3 & 189.9805 & 4.06 & 197.5483 & 8.21 & 182.5671 \\
\hline 4 & 318.189 & 5.53 & 328.2552 & 8.87 & 301.5048 \\
\hline 5 & 388.3815 & 5.20 & 402.7127 & 9.09 & 369.1714 \\
\hline 6 & 466.986 & 4.90 & 482.8012 & 8.45 & 445.1803 \\
\hline 7 & 561.6589 & 4.14 & 577.6732 & 7.11 & 539.3091 \\
\hline 8 & 686.9562 & 2.60 & 700.8135 & 4.69 & 669.5538 \\
\hline 9 & 821.6792 & 7.04 & 850.6059 & 10.81 & 767.6232 \\
\hline 10 & 985.4656 & 1.58 & 1003.5961 & 3.45 & 970.1321 \\
\hline
\end{tabular}

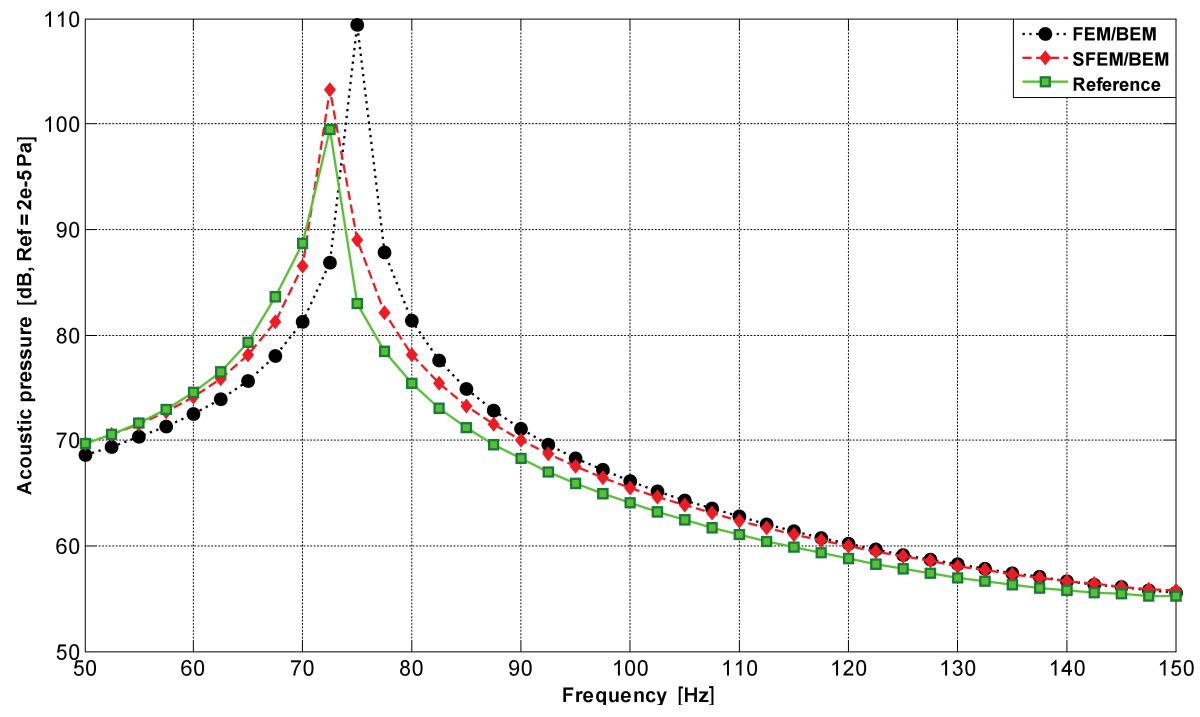

Fig. 9. The acoustic pressure frequency responses of the driver's seat point.

\section{Conclusions}

This paper presents novel numerical technique called SFEM/BEM to predict the structure-acoustic coupled problems, especially for shell structureacoustic coupled system. Some application examples are investigated in detail to study the panel eigenmodes and structure-acoustic coupled responses. The following conclusion can be derived.

1. The SFEM/BEM can well predict the shell-like structure performance, such as the eigenmodes, structure-acoustic coupled frequency responses. The results of the present method even can obtain higher accuracy than those of FEM/BEM method with the same coarse mesh. It indicates that the present method can be widely applied to solving structure-acoustic coupled problems without increasing much more computational time and decreasing the pre-processing time.
2. For the practical acoustic problems with complicated domains and boundary conditions, the SFEM/BEM obtains approximate accurate results with the coarse mesh. Due to the low demand of mesh density for pre-processing, the coupled SFEM/BEM can be widely applied to solving the vehicle structure-acoustics coupled problems.

3. In present work, the SFEM/BEM can only deal with the shell-like structure problems, but cannot solve the 3-dimensions solid structure problems. So the further researches are needed in future work.

\section{Acknowledgments}

This project is supported by National Natural Science Foundation of China (Grant No. 51605391) and the Chongqing science \&technology commission (CSTC) (No.2015JCYJA60008). 


\section{References}

1. Bathe K.J., Dvorkin E.N. (1985), A four-node plate bending element based on Mindlin/Reissner plate theory and a mixed interpolation, International Journal for Numerical Methods in Engineering, 21, 367-383.

2. Bathe K.J., Nitikitpaiboon C., Wang X. (1995), A mixed displacement-based finite element formulation for acoustic fluid-structure interaction, Computers \& Structures, 56, 2-3, 225-237.

3. Chen P.T., Ju S.H., ChA K.C. (2000), A symmetric formulation of coupled BEM/FEM in solving responses of submerged elastic structures for large degree of freedoms, Journal of Sound and Vibration, 233, 407-422.

4. Chог P. (1997), Application of a directly coupled boundary element and finite element model to the $d y$ namics of coupled acoustic silencers, $\mathrm{PhD}$ dissertation, North Carolina State University.

5. Coyette J.P. (1999), The use of finite-element and boundary-element models for predicting the vibroacoustic behaviour of layered structures, Advances in Engineering Software, 30, 133-139.

6. DAVIDSSON P. (2004), Structure-acoustic analysis: finite element modeling and reduction methods, $\mathrm{PhD}$ dissertation, Lund University.

7. Everstine G.C. (1981), A symmetric potential formulation for fluid-structure interaction, Journal of Sound and Vibration, 79, 1, 157-160.

8. Everstine G.C. (1997), Finite element formulations of structural acoustics problems, Computers \& Structures, 65, 3, 307-321.

9. Everstine G.C., Henderson F.M. (1990), Coupled finite element/boundary element approach for fluid structure interaction, The Journal of the Acoustical Society of America, 87, 5, 1938-1945.

10. FREDRIK H. (2001), Structure-acoustic analysis using $B E M / F E M$ : Implementation in MATLAB, Master Dissertation, Lund University.

11. Hamdi M.A., Ousset Y., Verchery G. (1978), A displacement method for the analysis of vibrations of coupled fluid \& structure systems, International Journal for Numerical Methods in Engineering, 13, 139150 .

12. He Z.C., Li G.Y. et al. (2013), An improved eigenfrequencies prediction for three-dimensional problems using face-based smoothed finite element method (FSFEM), Acta Mechanica Solida Sinica, 26, 2, 140-150.
13. He Z.C., LiU G.R. et al. (2011), A coupled ESFEM/BEM method for fluid-structure interaction problems, Engineering Analysis with Boundary Elements, 35, 140-147.

14. Jeans R.A., Mathews I.C. (1990), Solution of fluidstructure interaction problems using a coupled finite element and variational boundary element technique, The Journal of the Acoustical Society of America, 88, 2459-2466.

15. Jeans R.A., Mathews I.C. (1993), A unique coupled boundary element/finite element method for the elastoacoustic analysis of fliud-filled thin shell, The Journal of the Acoustical Society of America, 94, 3473-3479.

16. Kopuz S. (1996), Integrated FEM/BEM approach to the dynamic and acoustic analysis of plate structures, Engineering Analysis with Boundary Elements, 17, 269-277.

17. Li W., Chai Y.B. et al. (2014), Analysis of coupled structural-acoustic problems based on the smoothed finite element method ( $S$-FEM), Engineering Analysis with Boundary Elements, 40, 84-91.

18. Liu G.R., Dai K.Y., NGUYen T.T. (2007a), A smoothed finite element method for mechanics problems, Computational Mechanics, 39, 859-877.

19. LiU G.R., NGUYen T.T., DAI K.Y., LAM K.Y. (2007b), Theoretical aspects of the smoothed finite element method (SFEM), International Journal for $\mathrm{Nu}-$ merical Methods in Engineering, 71, 902-930.

20. Morand H., OHAYon R. (1979), Substructure variational analysis of the vibrations of coupled fluid structure systems- finite-element results, International Journal for Numerical Methods in Engineering, 14, 5, 741755 .

21. Nehete D.V., Modak S.V., Gupta K. (2015), Structural FE model updating of cavity systems incorporating vibro-acoustic coupling, Mechanical Systems and Signal Processing, 50-51, 362-379.

22. Tonga Z., Zhang Y., Zhang Z., Hua H. (2007), Dynamic behavior and sound transmission analysis of a fluid-structure coupled system using the direct$B E M / F E M$, Journal of Sound and Vibration, 299, 645-655.

23. Wang G., Cui X.Y., Liang Z.M., Li G.Y. (2015), $A$ coupled smoothed finite element method (S-FEM) for structural-acoustic analysis of shells, Engineering Analysis with Boundary Elements, 61, 207-217. 